\title{
Estrategia Didáctica Basada En La Lúdica Para La Adquisición De Competencias Financieras En Estudiantes De La Licenciatura En Administración
}

\author{
Armando Sánchez-Macías, PhD \\ Virginia Azuara-Pugliese, PhD \\ Ma Lourdes Martínez-Cerda, MSc \\ Universidad Autónoma de San Luis Potosí, México
}

Doi: 10.19044/esj.2018.v14n7p69 URL:http://dx.doi.org/10.19044/esj.2018.v14n7p69

\begin{abstract}
Financial competencies are about knowledge and understanding of both, financial concepts and financial risks, as well as the confidence to put them into practice. Ludic didactic strategies can be oriented towards knowledge acquisition of such skills. This paper aims to describe the impact of the use of a ludic didactic strategy to acquire financial competencies in undergraduate Management students in the CARAO -UASLP. This study is correlational, longitudinal with a quantitative approach that includes a quasiexperiment. A previous financial competencies diagnosis was performed as well as a latter one in an experimental and control group using a test and a survey. All of the students allocated in the second semester were tested and surveyed. The intervention done only in the experimental group was through a board game called "Cashflow 101" during a period of time of two months. The test outcomes show that most students require financial education and according to the survey approximately $70 \%$ has never used financial services altogether. Nevertheless, a relationship was found among financial competencies and various aspects of social contexts, such as owning a house or a car, which seem to increase the chances of having used financial services. Overall, it is clear that the use of the board game serves as a ludic tool for developing financial skills in a ludic form.
\end{abstract}

Keywords: Financial literacy, financial competencies, ludic didactic strategies, financial inclusion

\section{Resumen}

Las competencias financieras hacen referencia al conocimiento y comprensión de los conceptos y riesgos financieros, así como la confianza para aplicarlos. En este sentido, la lúdica como estrategia didáctica puede orientarse 
a la adquisición de saberes por lo que en este estudio es considerada como estrategia para la construcción de las competencias financieras. La presente investigación tiene como objetivo describir el impacto del uso de una estrategia didáctica basada en la lúdica para la adquisición de competencias financieras en estudiantes de la Licenciatura en Administración de la CARAO de la UASLP. Se realizó una investigación correlacional, longitudinal, con un enfoque cuantitativo, incluyendo un cuasiexperimento. Se aplicó un diagnóstico previo y posterior de las competencias financieras en un grupo experimental y otro de control mediante un test y un cuestionario. El muestreo fue de tipo censal y consideró a los estudiantes de Administración de $2^{\circ}$ semestre. La intervención consistió en el uso del juego de mesa Cashflow 101 (C) durante dos meses aplicado únicamente al grupo experimental. Los resultados del test muestran que la mayoría de los estudiantes requiere de educación financiera y que el $70 \%$ aproximadamente nunca ha usado los servicios financieros. Por otro lado, se encontró relación entre la variable competencias financieras y aspectos del contexto como tener casa propia y vehículo como factor que aumenta la probabilidad del uso de servicios financieros. Se puede decir que el uso de un juego como estrategia de aprendizaje-enseñanza puede considerarse una buena herramienta para el desarrollo de habilidades financieras.

Palabras-clave: Educación financiera, competencias financieras, estrategia didáctica-lúdica, inclusión financiera

\section{Introducción:}

El uso de productos y servicios financieros promueve el crecimiento económico en las economías emergentes y avanzadas; contribuye a reducir la pobreza, a fomentar el desarrollo sostenible y el progreso de la sociedad (Garcia, Grifoni , López, \& Mejía, 2013). Es por ello que es muy relevante conocer el nivel de competencias que poseen los individuos que utilizan los distintos servicios proporcionados por el sistema financiero formal; ya que, aunque la Asociación Mexicana de Bancos (ABM, 2013) afirma que el Sistema Financiero Mexicano (SFM) es sólido y está muy bien capitalizado, también es cierto que México se encuentra en el lugar número 45 entre las 78 economías de la muestra utilizada en el Reporte de Crecimiento y Desarrollo Inclusivo según el Índice de Inclusión y Desarrollo Económico (WEF, 2017).

En este sentido, el sistema bancario en México creció sus utilidades en el periodo de enero a julio de 2014 a 2015 cinco veces más que la economía del país en ese mismo periodo (CNBV, 2016). En 2017 se lograron utilidades récord con un crecimiento del $28.4 \%$ con respecto al año anterior (CNBV, 2018). A su vez la Comisión Federal de Competencia Económica (COFECE, 2014) menciona que el negocio de los servicios financieros en México está 
concentrado en siete grupos financieros que controlan el $70 \%$ del mercado, y que la falta de competencia crea obstáculos para que nuevos actores ofrezcan sus servicios extendiendo su alcance en lugares más allá de donde es altamente rentable. Es por ello que se afirma que la desigualdad económica y social en México tiene como una de sus causas a la exclusión financiera, así como la inequidad y pobreza, sumado a un lento crecimiento de zonas rurales en México (Álvarez, Páramo, \& Carpio, 2011).

A continuación, se abordan los términos que permiten contextualizar la presente investigación, siendo los conceptos centrales (inclusión, educación, cultura y competencias financieras) que influyen en la formación financiera del individuo, también se abordan las formas como se evalúan las competencias financieras en los individuos.

La inclusión financiera es definida como:

El proceso de promoción de un acceso asequible, oportuno y adecuado a una amplia gama de productos y servicios financieros regulados y la ampliación de su uso por todos los segmentos de la sociedad, a través de la aplicación de enfoques innovadores o existentes hechos a la medida, incluyendo actividades de sensibilización y de educación financiera, con el fin de promover el bienestar económico y la inclusión económica y social (G20 y OCDE, 2013, pág. 17).

Con ello se puede decir que la inclusión financiera es una necesidad imperante para México, al tener un efecto positivo sobre la pobreza y la desigualdad, propiciando el desarrollo económico y social. Según Clarke, Xu $\&$ Zou (2003) y Honohan (2007) un incremento del $10 \%$ en la disponibilidad y acceso a servicios financieros reduce en 0.6 puntos el coeficiente de Gini que mide la desigualdad en ingreso. Así como también un crecimiento del $10 \%$ en el crédito hacia los particulares disminuye la pobreza en casi el 3\%.

Según datos de la Encuesta Nacional de Inclusión Financiera 2013 y 2015 (ENIF), (INEGI y CNBV, 2012; 2015) aumentó el número de adultos que contrató un servicio financiero, siendo los de mayor demanda las cuentas de ahorro con un $36 \%$ en 2012 y de un $44 \%$ en 2015; las cuentas de crédito representaron un $27 \%$ en 2012 contra un $29 \%$ en 2015; en cuanto a seguros fue de un 22\% en 2012, mientras que en 2015 fue de un 25\%; en ahorros para el retiro en 2012 fue de un 28\%, mientras que en 2015 fue de $41 \%$. En el informe en materia de inclusión financiera, Global Findex 2014, se encontró que el $51 \%$ de los habitantes adultos (15 años o más) de América Latina y el Caribe tienen una cuenta bancaria, frente a un $39 \%$ en 2011, pero, aunque este avance es importante, todavía 210 millones de adultos siguen sin estar bancarizados (Banco Mundial, 2015).

Diversas experiencias nacionales e internacionales (Álvarez, Páramo, \& Carpio, 2011; Marshall, 2011; G20 y OECD, 2013; Garay, 2016; EIU, 2016; Martínez-Carrasco, Muñoz, Eid, \& Colino, 2016; Loungani, 2017; OXFAM, 
2017; WEF, 2017) dan cuenta del hecho de que para lograr incluir a los excluidos se requiere una ruta basada en la educación; es decir, la comunicación organizada y sustentada, que está diseñada para producir aprendizaje (INEGI, 2005); sin embargo, para muchos países no es una prioridad, sucediendo lo mismo en un ámbito más específico como lo es la educación financiera. ¿Y qué es esto? según la Organización para la Cooperación y el Desarrollo Económico (OCDE), la educación financiera se define como:

El proceso por el que los consumidores financieros/inversores mejoran su comprensión de los productos financieros, conceptos y riesgos y, a través de la información, la enseñanza y/o el asesoramiento objetivo, desarrollan las habilidades y confianza para adquirir mayor conciencia de los riesgos y oportunidades financieras, tomar decisiones informadas, saber dónde acudir para pedir ayuda y tomar cualquier acción eficaz para mejorar su bienestar financiero (OECD, 2005).

Por otro lado, El Banco del Ahorro Nacional y Servicios Financieros (BANSEFI) la define como una herramienta invaluable para promover la cultura del ahorro y ayudar a crear las condiciones que permitan una mayor inclusión financiera de las personas de escasos recursos. También menciona que la educación financiera favorece un mayor y mejor uso de los servicios financieros formales, lo que ofrece beneficios para los individuos, las instituciones financieras y la economía en su conjunto (BANSEFI, 2016).

En México la educación financiera ha sido relegada durante muchos años; mientras en otras naciones, desde hace décadas, se ha integrado este tipo de educación en los programas educativos, en nuestro país apenas se está analizando cómo introducirla a las escuelas. De acuerdo con datos de la Comisión Nacional para la Protección y Defensa de los Usuarios de Servicios Financieros, cerca de 62 de cada 100 mexicanos carece de educación financiera (CONDUSEF, 2010, par. 1). Con lo antes mencionado, se puede observar que éstas manifiestan el bajo conocimiento o el total desconocimiento que se tiene del sistema financiero, es decir, se desconocen los beneficios de los diferentes intermediarios y mercados financieros; a través de los cuales una variedad de instrumentos moviliza el ahorro hacia su uso más productivo, contribuyendo a elevar el crecimiento económico sostenido y el bienestar de la población (BANXICO, 2016, par. 2).

La educación financiera es indispensable no solamente para los asesores o personas dedicadas al sector financiero, sino que todos deben tener un nivel de educación en este tema, para contribuir a una mejor calidad de vida y tomar decisiones acertadas en el manejo de los recursos financieros, es decir, capacidad para desarrollar competencias financieras (INEE, 2013). Para comprender el concepto de competencia financiera se asume lo establecido por el Informe PISA de 2012: "La competencia financiera hace referencia al 
conocimiento y comprensión de los conceptos y riesgos financieros, y a las destrezas, motivación y confianza para aplicar dicho conocimiento y comprensión con el fin de tomar decisiones eficaces en distintos contextos financieros, mejorar el bienestar financiero de los individuos y la sociedad, y permitir la participación en la vida económica" (INED 21, 2014, par. 2).

La competencia financiera se considera actualmente como una pieza clave para lograr el desarrollo económico y financiero sostenido y estable en el mundo. En este sentido la Organización para la Cooperación y el Desarrollo Económico (OCDE) han establecido los Principios para las Estrategias Nacionales en Educación Financiera (OCDE, 2005a). Estudios sugieren que el nivel de competencias financieras está relacionado con su contexto familiar y educativo, es decir, las personas con mayores competencias provienen de familias con alto nivel educativo y que poseen diversidad de productos financieros (Raccanello \& Herrera, 2014).

Lo anterior contribuye a la formación de una cultura financiera, que se refiere a los hábitos y costumbres para administrar, incrementar y proteger el patrimonio (Amezcua García, Arroyo Grant, \& Espinosa Mejía, 2014). La falta de esta ha traído consigo múltiples preocupaciones para los países desarrollados debido al "efecto crisis"; ya que se considera que lleva a los ciudadanos a tomar decisiones inadecuadas. Aunque también, existen otros factores como lo son el incremento de riesgos y decisiones financieras, así como el aumento de la oferta y demanda de una amplia gama de productos y servicios financieros, siendo estos los factores clave que justifican la importancia de las competencias financieras (OECD, 2012).

\section{Estrategia didáctica mediante la lúdica}

Todos los procesos educativos -como las acciones para la construcción de competencias financieras- necesitan, para su desarrollo, de estrategias de enseñanza y de aprendizaje (Torres Maldonado \& Girón Padilla, 2009). La implementación de dichas estrategias se logra mediante el uso de la didáctica (Medina Riilla \& Salador Mata, 2009), es decir, las formas y métodos mediante los cuales se procura el desarrollo de las competencias en las persona, Velazco \& Mosquera (2010, págs. 1-2) mencionan que: el concepto de estrategias didácticas se involucra con la selección de actividades y prácticas pedagógicas en diferentes momentos formativos, métodos y recursos en los procesos de Enseñanza-Aprendizaje.

Existe suficiente documentación que respalda la “...correlación significativa (...) entre el juego, el aprendizaje, los procesos de pensamiento, la sociabilidad y el equilibrio emocional" (Domínguez, 2015, pág. 9). El juego se cristaliza en la lúdica, la cual es definida en este trabajo como una dimensión del desarrollo del individuo que incluye la necesidad humana de la comunicación, de sentir, de expresarse y de producir en las demás emociones 
orientadas hacia el entretenimiento y el esparcimiento. La lúdica "fomenta el desarrollo psicosocial, la conformación de la personalidad, la evidencia de valores y puede orientarse a la adquisición de saberes..." (Gómez, Molano, \& Rodríguez, 2015, pág. 29).

La lúdica se constituye como una estrategia de aprendizaje significativo; es decir, que los nuevos aprendizajes conectan con los anteriores (Ausubel, Novak, \& Hanesian, 2009), permitiendo la comunicación, cooperación, socialización y el respeto entre los sujetos; al tiempo que permite la construcción activa de aprendizaje, la cual supone que la elaboración del conocimiento se debe llevar a la luz de la acción y las prácticas, pues sólo se aprende bien lo que se puede experimentar, analizar, comprender y vincular con las prácticas concretas mediante la experiencia (Caballero, 2010, pág. 164).

Una persona sin competencias financieras no puede tomar decisiones apropiadas para sacar el máximo provecho de las herramientas que ofrecen las instituciones bancarias, de seguros, bursátiles, financieras, entre otras. Asimismo, no está en posibilidad de entender qué es lo que más le conviene para consolidar y proteger su patrimonio contra los riesgos y prácticas abusivas o faltas de ética en el sistema.

En la generación digital actual, la lúdica se ha convertido en una táctica popular para fomentar conductas específicas y aumentar la motivación y el compromiso. Aunque se encuentra comúnmente en las estrategias de mercadotecnia, ahora se está implementando también en muchos programas educativos, ayudando a los educadores a encontrar el equilibrio entre el logro de sus objetivos y la satisfacción de las cambiantes necesidades de los estudiantes (Huang \& Soman, 2013).

El concepto de lúdica puede ser simplificado en los siguientes pasos (ver figura 1): 


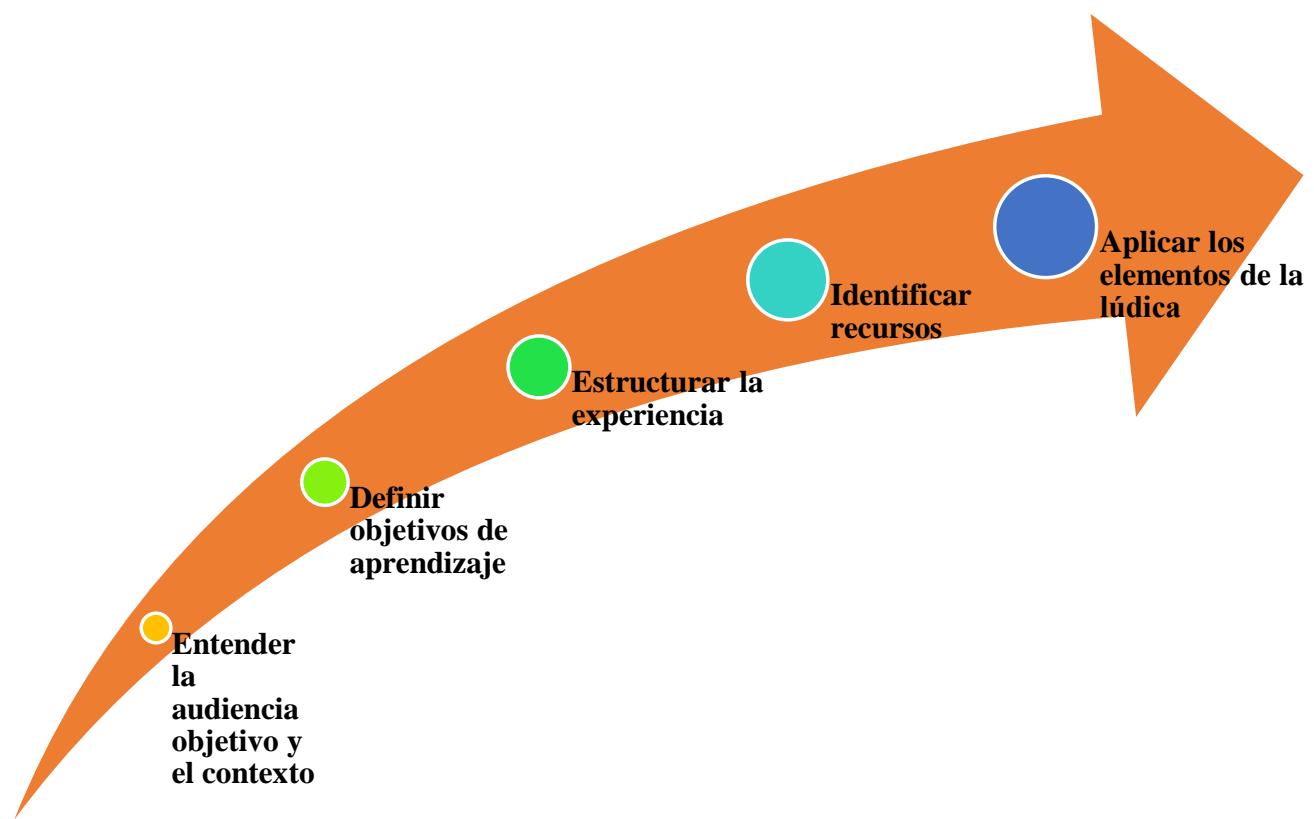

Figura 1. Proceso simplificado de la lúdica Adaptado de: Huang \& Soman (2013)

En el primer paso, denominado como "Entender la audiencia objetivo y el contexto", se refiere a los puntos de dolor, los cuales se definen como aquellos factores que impiden a los estudiantes a avanzar a través del programa de aprendizaje y/o lograr los objetivos; y que, dependen de factores como la edad del estudiante, el contexto y el campo, como pueden ser: la motivación, orgullo, habilidades, concentración, factores físicos, mentales y emocionales; y el ambiente de aprendizaje y la naturaleza del curso.

En el segundo, mientras tanto, se establece la necesidad que el instructor defina los objetivos de aprendizaje que se busca que el estudiante consiga al terminar el programa. Existen diferentes tipos de objetivos: objetivos generales de instrucción, las cuales pueden incluir hacer que el estudiante complete una tarea, realizar un examen o un proyecto; objetivos de aprendizaje específico, los cuales pueden incluir que el estudiante comprenda un concepto, que sea capaz de realizar una tarea después del entrenamiento o que complete el programa de aprendizaje; y objetivos de comportamiento, que pueden requerir que el alumno se concentre en clase, complete las tareas más rápido, minimice las distracciones en clase, entre otros.

En el tercero, "Estructurar la experiencia", en el cual incluyen las etapas y fases como herramientas poderosas, ya que permiten a los instructores secuenciar el conocimiento y cuantificar lo que los estudiantes deben aprender y lograr al final de cada etapa o fase. Estos, además, funcionan también para los estudiantes, ya que hacen que el objetivo final parezca más alcanzable y 
medible, a la vez que aseguran que los obstáculos dentro y entre cada etapa sean fácilmente identificables.

En el cuarto punto se deben "Identificar recursos"; es decir, una vez que se han definido las fases o etapas del programa, el instructor puede juzgar de manera más sencilla cuáles etapas pueden ser ludificadas y cómo. Esto es exactamente lo que hace que los juegos sean atractivos, ya que los estudiantes reciben retroalimentación rápida si hacen una tarea incorrecta y tienen la oportunidad de volver a intentarlo.

Finalmente, en el último punto hay que "Aplicar los elementos de la lúdica", para lo cual se utiliza el proceso de ludificación en la educación, el cual, se reduce a la adición de elementos similares a los juegos, también llamados mecánicas de juegos, en entornos que no son de juego. La mecánica del juego se puede clasificar como: elementos propios, que pueden ser puntos, insignias de logro, niveles o simplemente restricciones de tiempo. Estos elementos hacen que los estudiantes se concentren en competir contra sí mismos y reconocer el auto-logro; elementos sociales, como la competencia interactiva o cooperativa (como lo son las tablas de clasificación), estos elementos ponen a los estudiantes en comunidad con otros estudiantes, y su progreso y los logros se hacen públicos.

Se ha referido hasta aquí el proceso para implementar la lúdica, su funcionalidad y los elementos a evaluar cuando de competencias financieras, la relación que tienen con la inclusión, la cultura financiera y el contexto, se trata. A continuación, se referirá a la herramienta didáctica lúdica en lo particular.

\section{La aportación de Kiyosaki}

El juego Cashflow 101 (c) (desarrollado por el autor, inversionista y empresario Robert Kiyosaki), es un juego de mesa educativo que aborda la contabilidad, finanzas y estrategias de inversión, para que se utilicen conceptos contables de una forma amigable. Se aplican operaciones básicas como sumar y restar, así como evaluar constantemente la situación financiera durante el juego, con la finalidad de identificar la información relevante antes de realizar un movimiento.

El objetivo principal del juego consiste en lograr ser financieramente libre; es decir, salir de la denominada "carrera de la rata", la cual simula la vida de un trabajador con un sueldo fijo asignado y con el riesgo de ser despedido y que en el juego está representada en el círculo interior del tablero; esto se consigue al aumentar los ingresos pasivos hasta superar los gastos mensuales; ya que, como menciona TRA Profesional Services (2012), la libertad financiera consiste generalmente en un $80 \%$ de actitud mental y un $20 \%$ de aspectos técnicos y pasar a la "vía rápida", que es la pista exterior del tablero y representa a la vida de los ricos. 
El juego incluye tarjetas de juego referentes al empleo, el estado financiero (ingresos y gastos) y el día de pago; así como otras referentes a las inversiones y el mercado que funcionan en conjunto con determinadas casillas, las cuales señalan que al caer en una de ellas se debe tomar una tarjeta que puede ser: pequeño negocio, gran negocio, mercado y capricho. Además, considera casillas con aspectos como la reducción de personal (es decir, ser despedido), beneficencia (que promueve la generosidad) y la inclusión de un nuevo integrante en la familia (tener hijos) (Fridman, 2010).

El juego cashflow ha sido implementado alrededor del mundo principalmente en clubs de Argentina, España y Estados Unidos; como menciona Fridman, el juego es practicado por aficionados a los libros de autoayuda financiera. Al jugar en un club los participantes pueden conectarse con gente que tiene inquietudes e intereses similares, como lo es la libertad financiera (Fridman, 2016).

Como menciona Fridman (2016) "No es un juego. Es una herramienta para acondicionar la cabeza para las finanzas, la contabilidad y los negocios", por lo que se debe de tomar con seriedad y, ya sea por cuenta propia o con la ayuda de otros obtener una transformación de sí mismos con el fin de alcanzar cierto estado de felicidad, pureza, sabiduría o inmortalidad al evaluar su yo financiero o, como lo refiere Foucault (2008), tecnologías del yo.

Kiyosaki (2015) se dirige fundamentalmente a gente que trabaja en relación de dependencia, considera que existe un cuadrante del flujo de dinero (empleado, auto empleado, dueño e inversionista) en las que se requiere de diferentes estructuras de pensamiento, aptitudes técnicas, caminos educativos y distintos tipos de personalidades.

No importa la cantidad de dinero que se tenga, sino los ingresos pasivos que se es capaz de generar, es decir, no se gana por acumular dinero, sino por generar suficiente ingreso pasivo para pagar sus gastos de vida y así quedar financieramente libre.

\section{Algunas experiencias del uso de la lúdica en la educación}

Es pertinente analizar diferentes estudios de la implementación de estrategias de aprendizaje-enseñanza, en base a la lúdica en el campo financiero y económico para analizar sus alcances y su funcionalidad, por lo que se presentan algunos de ellos a continuación:

En un estudio realizado por Liu y otros (2011), se encontró que los jugadores se sentían satisfechos con la nueva forma de aprender (el juego) en un rango de satisfacción entre 3.30 a 4.00 donde 4.00 correspondía con "de acuerdo" además en la pregunta "piensas que el juego es una pérdida de tiempo para aprender finanzas los resultados se encuentran muy cercanos al indicar 2.30 que está muy cercano al valor de "desacuerdo" con puntuación de 2.00. Entre los principales temas que abarca el juego Second LifeC destaca la 
administración del dinero, la planeación financiera, la inversión básica, seguros, residencia y elección de carrera.

Por otra parte, Brennan y Vos (2013) señalan que un juego de simulación es un buen medio para aprender sobre los conceptos cuantitativos y financieros debido a dos argumentos: en primer lugar, porque un juego de simulación es una experiencia de aprendizaje atractiva en la que los estudiantes se absorben; y en segundo, porque los estudiantes encuentran más fácil adquirir conceptos numéricos y financieros cuando estos se contextualizan en una experiencia simulada del mundo real.

Además, se creó un curso similar a un juego llamado JFDI Academy®, el cual, mediante la aplicación de mecánica de juego a un curso introductorio de programación basado en esquemas tradicionales, se indujo un comportamiento constante de los estudiantes y se identificaron brechas en el progreso de aprendizaje de cada alumno. Además, los estudiantes que normalmente se inscriben en este curso alternativo están más motivados y buscan cursos de nivel avanzado en la asignatura (Huang \& Soman, 2013).

Deloitte Leadership Academy desarrolló, a su vez, una capacitación corporativa, en la que aplicó con éxito una de las mecánicas de ludificación más comunes - por puntos e insignias - a su programa de capacitación ejecutiva digital. Además, como los estudiantes eran ejecutivos experimentados, los puntos importantes que debían abordarse eran que los estudiantes tenían tiempo limitado y el curso en sí tenía que ser de alto valor educativo (Dominguez, 2014).

Otro ejemplo es PlayMoolah(C) un programa de alfabetización financiera dirigido a niños y adultos jóvenes, lanzado en abril de 2012 por Min Xuan Lee y Audrey Tan, de la Universidad de Stanford. Con PlayMoolah@, el objetivo de los fundadores era alentar a las personas a tomar medidas con su comprensión de los conceptos financieros. Con este programa, el estudiante puede aprender de manera intercambiable los diferentes aspectos de: ganar, gastar, ahorrar, invertir y dar en un mundo de ensueño. Además, tenía que ser fácil navegar por el programa, para que pudieran aprender por sí mismos, mientras que los padres monitoreaban y guiaban su progreso con la finalidad de evitar que perdieran el enfoque (Singapore Press Holdings, 2015).

\section{Metodología}

El enfoque de esta investigación sigue las recomendaciones de la OCDE (2005) en tanto que la educación financiera debe comenzar en la escuela, debido a que las personas deben de ser educadas en el tema lo más pronto posible en sus vidas. El contar con un grado efectivo de educación financiera es importante dado el creciente alcance y la accesibilidad de los productos y servicios financieros disponibles (Cameron, Calderwood, Cox, Lim, \& Yamaoka, 2013). A su vez esta investigación se aleja de la concepción 
instrumentalista de la educación financiera de Cole et. al. (2009); Servon \& Kaestner (2008) y CGAP (2005) en Birochi \& Pozzebon (2016, pág. 268) como "...promotora de eficiencia y eficacia del sistema financiero a través de la corresponsabilidad de los consumidores (derechos y obligaciones)". Se asume luego la concepción transformativa o crítica con un enfoque humanitario y social en el que los individuos con grandes limitaciones socioeconómicas logran desarrollarse al fortalecer sus capacidades individuales, empoderamiento y autonomía (Johnston \& Maguire, 2005; Landvogt, 2006; Fernando, 2006; Burkett \& Sheehan, 2009; Sempere, 2009; Mayoux, 2010; Augsburg \& Fouillet, 2010 y Cabraal, 2011 en Birochi \& Pozzebon 2016).

El objetivo de la presente investigación considera implementar el juego Cashflow 101@ que se ha hecho muy popular entre emprendedores, para describir el impacto del uso de una estrategia didáctica basada en la lúdica -el uso del juego Cashflow 101@- en la adquisición de competencias financieras en estudiantes, identificar cuáles son las mejores prácticas de su uso y sus posibles implicaciones en el uso y percepción de los servicios financieros.

Este trabajo presenta una investigación de tipo cuantitativa, descriptiva-correlacional y longitudinal. Se realizó un diagnóstico inicial a dos grupos (experimental y de control) mediante un test y un cuestionario, posteriormente se realiza la intervención en el grupo experimental, para finalmente volver a medir en ambos grupos.

La intervención implica el uso del juego de mesa Cashflow 101@ durante dos meses por parte del grupo experimental. Esta investigación se constituye en un cuasiexperimento dado que, aunque se cuenta con grupo de control, ambos grupos estaban previamente formados. El muestreo es de tipo censal.

Se diseñó un test acorde a dos de las tres dimensiones de la competencia financiera (contenido, procesos y contexto) consideradas en el Programa para la Evaluación Internacional de Estudiantes (PISA, 2012), por sus siglas en inglés (Programme for International Student Assessment).

Cada uno de dichos ámbitos se subdivide, además, en cuatro líneas de análisis. Comenzando con los contenidos, se tienen los siguientes bloques (OCDE, 2012).

- $\quad$ El dinero y las transacciones.

- La planificación y la gestión de las finanzas.

- $\quad$ El riesgo y las compensaciones.

- $\quad$ El mundo de las finanzas.

Por su parte, los procesos se subdividen en las siguientes estrategias:

- Identificar la información financiera.

- Analizar la información en un contexto financiero. 
- Evaluar cuestiones financieras.

- Aplicar el conocimiento y la comprensión financiera.

Finalmente, los contextos se subdividen en las siguientes situaciones:

- $\quad$ La educación y el trabajo.

- El hogar y la familia.

- $\quad$ El individuo.

- La sociedad.

Por su parte la dimensión contexto se midió mediante una encuesta que, además de analizar los factores socioeconómicos, identificó la frecuencia de uso y las percepciones de los servicios financieros. La población para el estudio de investigación fueron los alumnos de la Licenciatura en Administración que actualmente cursan $2^{\circ}$ semestre de la Coordinación Académica Región Altiplano Oeste (CARAO) de la Universidad Autónoma de San Luis Potosí (UASLP).

\section{Resultados parciales y discusión previa}

Los alcances del presente artículo incluyen el diagnóstico de los resultados preliminares que a continuación se presentan, al momento de escribir este documento el experimento aún se encontraba en proceso. Los sujetos de estudio de la presente investigación son los estudiantes de Licenciatura de Administración que actualmente cursan $2^{\circ}$ semestre en la UASLP, siendo entre ambos grupos un total 44 estudiantes, de los cuales, un $39 \%$ son hombres y $61 \%$ mujeres. De éstos, el $98 \%$ señaló ser soltero y el $2 \%$ restante casado; además, se decidió incluir una pregunta sobre si se tenía dependientes económicos, a lo que un $87 \%$ respondió que sí, un $11 \%$ señaló no tener ninguno, y el restante $2 \%$ no contestó. Asimismo, un $60 \%$ de 10 s alumnos indicó vivir en casa propia y un $40 \%$ indicó que no, así como un $48 \%$ tiene vehículo y el 52\% indicó que no.

Para la evaluación de las percepciones respecto al sistema financiero y la adopción de los servicios que este ofrece a los usuarios se obtuvieron los siguientes resultados:

Los encuestados consideran que el servicio en sucursales bancarias, cajeros automáticos, así como el servicio de la banca electrónica es bueno en promedio en un $50 \%$, y en un $40 \%$ regular, tal como se puede observar en la figura 2 siendo considerado el servicio brindado bueno con un mayor porcentaje de acuerdo con los resultados. 


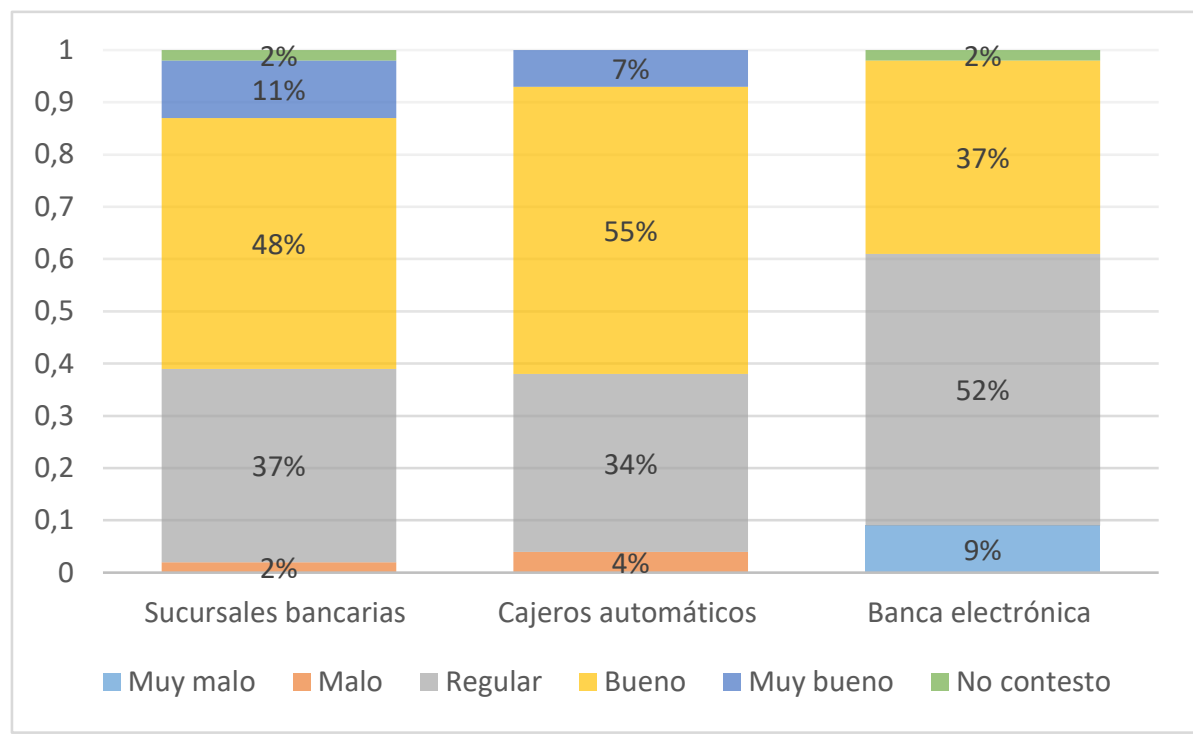

Figura 2.- Percepción de los servicios del sistema financiero Fuente: elaboración propia

Con el objetivo de conocer la percepción de los estudiantes respecto al sistema financiero, se formularon algunas afirmaciones donde los encuestados indicarían que tan de acuerdo están con ella en una escala del 1 al 5 donde 1 es total desacuerdo y 5 totalmente de acuerdo.

Respecto al uso de las cuentas bancarias para ahorrar el $48 \%$ está totalmente en desacuerdo; en cuanto a la respuesta respecto: a) a la confiabilidad de aseguradoras; b) a la confianza y seguridad de los depósitos de cuentas bancarias; c) a que las instituciones cumplen con lo que prometen en relación a las condiciones de crédito $\mathrm{y}, \mathrm{d}$ ) a que los bancos buscan constantemente mejorar sus productos y servicios para obtener la preferencia de sus usuarios, los resultados se concentran en ni de acuerdo ni en desacuerdo con un 50\% aproximadamente mostrando que no existe una opinión clara al respecto y/o que existe desconocimiento del servicio financiero. Se muestra en la figura 3. 


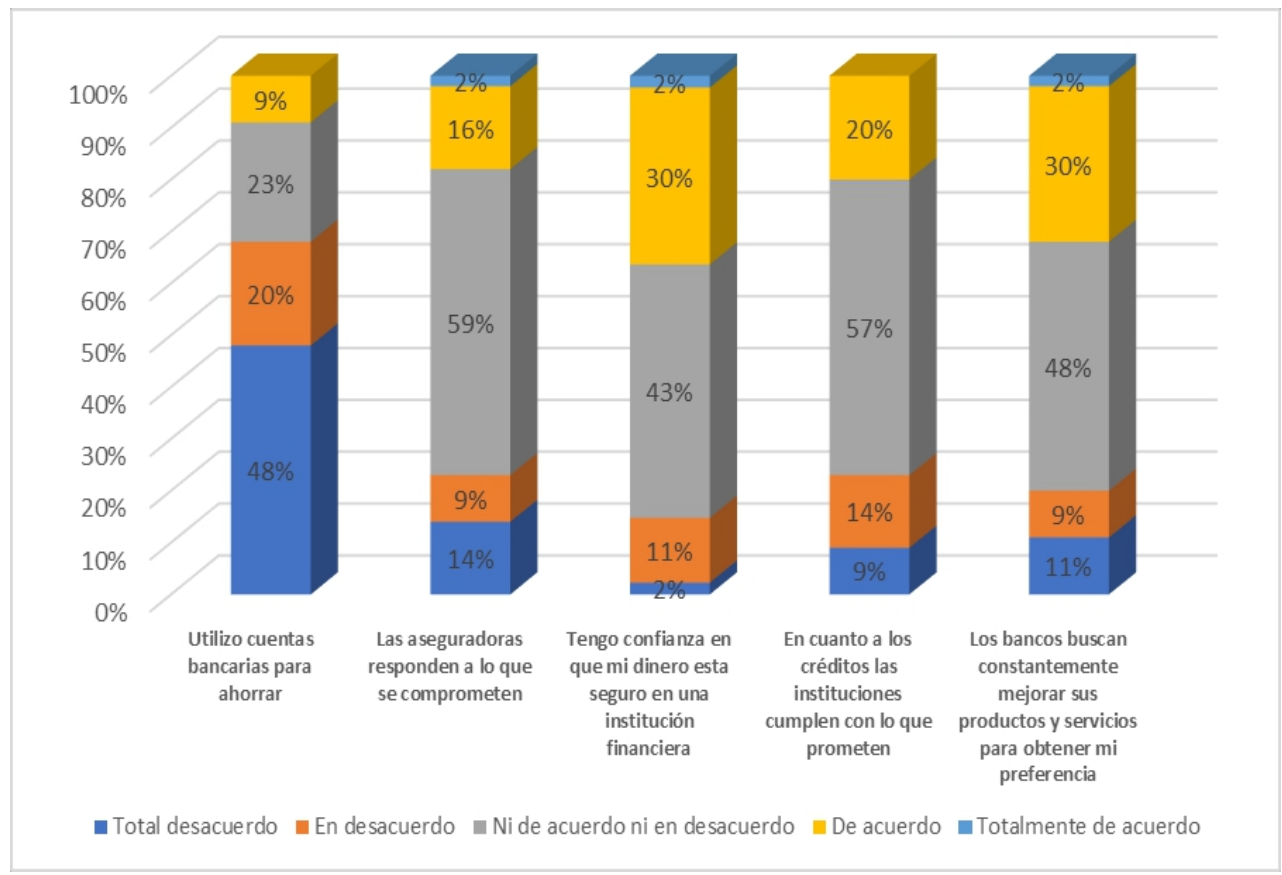

Figura 3. Percepción del sistema financiero

Fuente: elaboración propia

Por otra parte, también se hizo un análisis acerca de la frecuencia de uso de los servicios financieros, si se observa la figura 4, en promedio el $70 \%$ de los encuestados nunca ha utilizado algún servicio financiero; por otro lado, solo el $2 \%$ de los encuestados siempre hace uso de los corresponsales bancarios, siendo mínimo el porcentaje de los cuales lo utilizan con frecuencia; con 5\% el uso de seguros y $7 \%$ el uso de la terminal para recibir tarjetas (TPV). Vale la pena considerar el contraste que hay con los resultados, siendo buena la percepción que se tiene del servicio financiero, sin embargo, no se logra tener una idea clara al respecto dada la poca frecuencia de uso, es decir, es difícil evaluar objetivamente lo que no se conoce. 


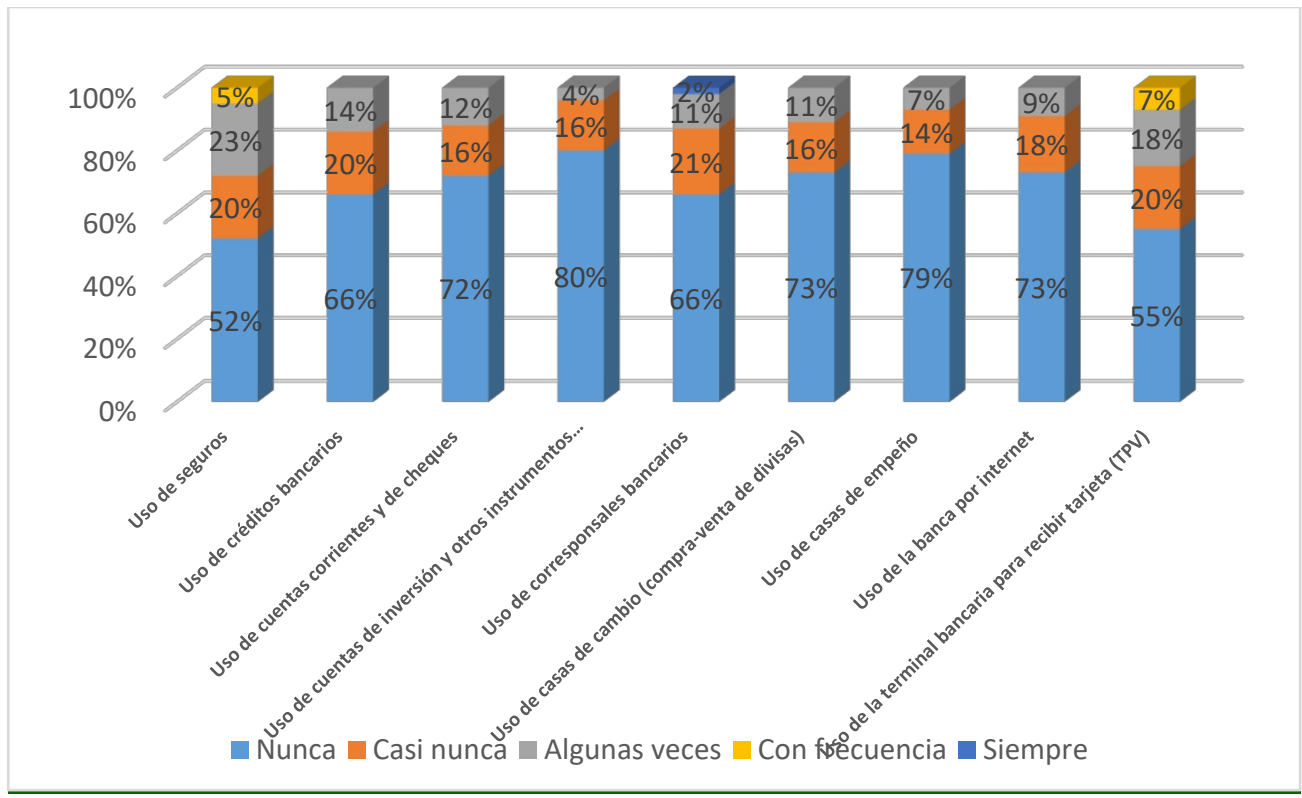

Figura 4. Frecuencia de uso de servicios financieros

Fuente: elaboración propia

En cuanto a los descriptivos DEL resultado de la prueba de competencias financieras en los grupos son los siguientes (ver tabla 1 y 2 ):

Tabla 1.- Resultados descriptivos de la aplicación del test

\begin{tabular}{|c|c|c|c|c|c|c|c|}
\hline \multirow[b]{3}{*}{ TIPO DE GRUPO } & & \multicolumn{6}{|c|}{ Casos } \\
\hline & & \multicolumn{2}{|l|}{ Válido } & \multicolumn{2}{|c|}{ Perdidos } & \multicolumn{2}{|c|}{ Total } \\
\hline & & $\mathrm{N}$ & Porcentaje & N & Porcentaje & N & Porcentaje \\
\hline \multirow{2}{*}{$\begin{array}{l}\text { Resultado test } \\
\text { competencias financieras }\end{array}$} & Experimental2a & & $100.0 \%$ & 0 & $0.0 \%$ & 27 & $100.0 \%$ \\
\hline & Control & & $100.0 \%$ & 0 & $0.0 \%$ & 17 & $100.0 \%$ \\
\hline
\end{tabular}

\begin{tabular}{|c|c|c|c|}
\hline \multicolumn{3}{|c|}{ TIPO DE GRUPO } & Estadístico \\
\hline \multirow{12}{*}{$\begin{array}{l}\text { Resultado test competencias } \\
\text { financieras }\end{array}$} & \multirow{6}{*}{$\begin{array}{c}\text { Experimental } \\
2 \mathrm{a}\end{array}$} & Media & 13.1111 \\
\hline & & Mediana & 13.0000 \\
\hline & & Desviación estándar & 1.717118 \\
\hline & & Mínimo & 9.00 \\
\hline & & Máximo & 16.00 \\
\hline & & Rango & 7.00 \\
\hline & \multirow{6}{*}{ Control } & Media & 12.2353 \\
\hline & & Mediana & 12.0000 \\
\hline & & Desviación estándar & 2.41168 \\
\hline & & Mínimo & 8.00 \\
\hline & & Máximo & 16.00 \\
\hline & & Rango & 8.00 \\
\hline
\end{tabular}


Como se observa la media en el grupo experimental es de 13.11 en una escala del 1 al 20. En el caso del grupo de control la media fue de 12.23 en una escala del 1 al 20. En ambos casos la máxima calificación fue de 16.

Dado lo anterior, al evaluar los resultados del test, se obtuvieron los siguientes resultados (ver figura 5).
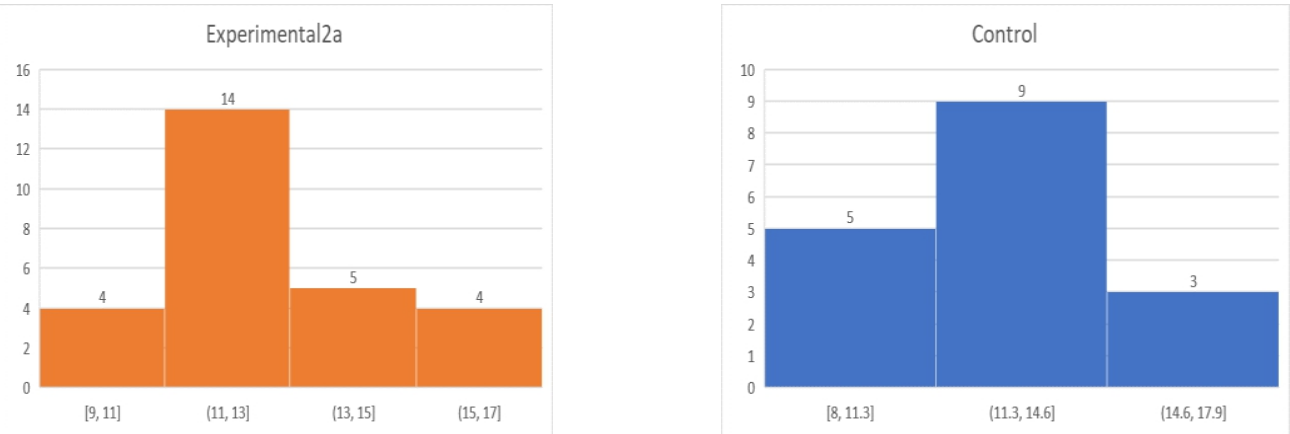

Figura 5.- Histogramas de los resultados del test en ambos grupos

Fuente: elaboración propia

Dado que el tamaño de las muestras es menor a 50 en ambos grupos, se considera el resultado de la prueba Shapiro-Wilk. Se encontró que el grupo experimental no tiene una distribución normal, mientras que el de control sí. Esto implicará utilizar pruebas estadísticas diferenciadas para el análisis de los pares de resultados (datos apareados) con la prueba U de Mann-Whitney en el grupo experimental y, la prueba t de Student en el grupo de control. Esto siempre y cuando la distribución de los datos en la segunda medición sea consistente con la primera.

Tabla 2.- Resultados descriptivos de la aplicación del test

\begin{tabular}{|c|c|c|c|c|c|c|c|}
\hline \multirow{2}{*}{ TIPODE GRUPO } & & \multicolumn{3}{|c|}{ Kolmogorov-Sminov $v^{2}$} & \multicolumn{3}{|c|}{ Shapiro-Wilk } \\
\hline & & Estadístico & $\mathrm{gl}$ & Sig. & Estadístico & $\mathrm{gl}$ & Sig. \\
\hline \multirow{2}{*}{$\begin{array}{l}\text { Resultado test } \\
\text { competencias financieras }\end{array}$} & Experimental2a & .215 & 27 & .002 & .914 & 27 & .028 \\
\hline & Control & .167| & 17 & $.200^{\circ}$ & .952 & 17 & .489. \\
\hline
\end{tabular}

Actualmente se está realizando la intervención, para posteriormente volver a revisar los resultados y mediante el uso de la prueba estadística t de Student -si la distribución de los resultados del test es normal- o de la prueba U de Mann-Whitney -si resulta no distribuirse de manera normal- establecer si la intervención realizada influyó en la probable variación de niveles de competencias financieras.

La estadística inferencial estudia cómo obtener conclusiones generales para toda la población a partir del estudio de una muestra, y el grado de fiabilidad o significado de los resultados obtenidos; en la presente investigación se realizaron algunas pruebas para inferir sobre el nivel de 
competencias financieras en los estudiantes de la CARAO, de acuerdo con los resultados de la muestra.

La prueba exacta de Fisher es el análisis de varianzas de más de dos grupos, que tiene su funcionamiento en relación con la variación explicada por las diferencias entre grupos y la variación individual, en sí el test exacto de Fisher permite analizar si dos variables dicotómicas están asociadas (Pértega Díaz \& Pita Fernández, 2004). Para medir las implicaciones del contexto (variables sociodemográficas) en el resultado del primer test se utilizó esta herramienta (para establecer relaciones entre variables cualitativas), probando relaciones estadísticamente significativas, se mencionan a continuación:

En cuanto al uso de créditos bancarios, hay mayor probabilidad de utilizarlos cuando se vive en casa propia con un índice de significancia de 0.020 y en cuanto si tienen vehículo la significancia es de igual manera 0.020 , así mismo si se tiene vehículo también hay mayor probabilidad del uso de corresponsales bancarios con 0.023 de significancia.

Respecto, al uso de cuentas de inversión y otros instrumentos financieros cuando se tiene dependientes económicos se relaciona con un 0.014 de significancia, y en cuanto al uso de terminal bancaria para recibir tarjeta hay mayor probabilidad si se vive en casa propia con 0.018 por lo que estas variables están significativamente relacionadas.

\section{Conclusion}

Los resultados del test muestran que la mayoría de los estudiantes requiere de educación financiera obteniendo como media 13 y 12 aciertos, en el grupo experimental y de control respectivamente, representando un $65 \%$ y $60 \%$. Se concluye que poseen conocimientos y habilidades en un nivel básico; se considera así dado el nivel universitario en el que se encuentran y las asignaturas impartidas en la institución acorde al plan de estudios. Respecto a los resultados obtenidos en el cuestionario del uso y percepción del servicio financiero se encontró que el $70 \%$ aproximadamente nunca ha usado los servicios financieros, siendo un posible factor para que no se evalúe objetivamente el sistema financiero, al mencionar que los servicios brindados son buenos en un $50 \%$ aproximadamente y en un $40 \%$ regular; es un alto porcentaje considerando que no se usan con frecuencia. Por otro lado, se encontró relación entre la variable competencias financieras con la dimensión contexto: vivir en casa propia y tener vehículo -más altos ingresos- aumenta la probabilidad del uso de servicios financieros, así como respecto al uso de cuentas de inversión y otros instrumentos financieros cuando se tiene dependientes económicos -necesidad de trabajar-. Esto es consistente con los datos ya expuestos de la ENIF (INEGI y CNBV, 2012; 2015).

Esta investigación permite detectar oportunidades de mejora pues hasta este punto evidencia que los estudiantes no solo no están familiarizados con el 
sistema financiero, sino que no están facultados y empoderados financieramente para hacer un uso adecuado de los servicios y productos que ofrece el sistema formal. Se asume la necesidad de una intervención que desarrolle competencias financieras para tomar decisiones de manera consciente y eficiente, y que esto a su vez, les permita crecer y contribuir al desarrollo económico no solo personal sino también al de su entorno. Se espera que la implementación que actualmente se hace de la estrategia didáctica lúdica pueda tener un impacto positivo en las competencias de los estudiantes, orientando a futuro su incorporación regular en los cursos de finanzas.

Tomando en consideración los estudios y prácticas que se han implementado como estrategias para el desarrollo de conocimientos en base al juego se tiene que existe suficiente documentación que respalda la relación entre el juego y el aprendizaje en específico como se mencionaba en el ámbito financiero económico. Se agradece el financiamiento del Fondo de Apoyo a la Investigación (FAI) convenio número C17-FAI-06-05.05 de la Universidad Autónoma de San Luis Potosí, para la realización de la presente investigación.

\section{References:}

1. ABM. (2013). La banca en México: compromiso social y desarrollo sustentable. México: Asociación de Bancos de México.

2. Álvarez, L. d., Páramo, T., \& Carpio, J. J. (2011). De la inclusión financiera a la intervención social: Una experiencia en el sureste de México. Nova Scientia, vol. 4, núm. 7, noviembre-abril 2011, pp. 125152.

3. Amezcua García, E. 1., Arroyo Grant, M. G., \& Espinosa Mejía, F. (2014). Contexto de la educación finanicera en México. Veracruz: Autor.

4. Amezcua García, E. 1., Arroyo Grant, M. G., \& Espinosa Mejía, F. (2014). Contexto de la educación finanicera en México. Veracruz.

5. Ausubel, D., Novak, J., \& Hanesian, H. (2009). Psicología Educativa, un punto de vista cognoscitivo. México: Trillas.

6. Banco Mundial. (2016). Global Findex 2016. Nueva York, EUA: Banco Mundial.

7. BANSEFI. (03 de Abril de 2016). Bansefi y la Educación Financiera. Obtenido de Bansefi y la Educación Financiera: https://www.gob.mx/bansefi/documentos/bansefi-y-la-educacionfinanciera

8. BANXICO. (2016). Sistema Financiero. Obtenido de Sistema Financiero: http://www.banxico.org.mx/divulgacion/sistemafinanciero/sistema-financiero.html 
9. Brennan, R., \& Vos, L. (2013). Effects of Participation in Simulation Game on Marketing Students' Numeray and Financial Skills. Journal of Marketing Education, 260.

10. Caballero, A. (2010). El juego, un recurso invaluable. México: Fuentes.

11. Cameron, M. P., Calderwood, R., Cox, A., Lim, S., \& Yamaoka, M. (2013). Factors Associated with Financial Literacy among High School Students. (pág. 3). Hamilton, New Zealand: University of Waikato.

12. Clarke, G., Xu, L. C., \& Zou, H.-f. (2003). Finance and income inequality: test of alternative theories. Washington D.C.: The World Bank.

13. CNBV. (2016). Comisión Nacional Bancaria y de Valores. Obtenido de Información Estadística: http://www.cnbv.gob.mx/SECTORESSUPERVISADOS/BANCA-

MULTIPLE/Paginas/Informaci\%C3\%B3n-Estad\%C3\%ADstica.aspx

14. CNBV. (2018). Comisión Nacional Bancaria y de Valores. Obtenido de Información de la situación financiera: banca múltiple: http://www.cnbv.gob.mx/Paginas/PortafolioDeInformacion.aspx

15. COFECE. (2014). Trabajo de investigación y recomendaciones sobre las condiciones de competencia en el sector financiero y sus mercados. México: Comisión Federal de Competencia Económica.

16. CONDUSEF. (05 de Octubre de 2010). CNNExpansión. Obtenido de CNNExpansión: http://expansion.mx/mi-dinero/2010/10/05/conduseftras-la-educacion-financiera?internal_source $=$ PLAYLIST

17. Domínguez, M. T. (2015). La lúdica una estrategia pedagógica depreciada| . Ciudad Juárez: Universidad Autónoma de Ciudad Juárez.

18. EIU. (2016). Microscopio Global 2016. Análisis del entorno para la inclusión financiera. Nueva York, EUA: Economist Intelligence Unit. The Economist.

19. Foucault, M. (2008). Tecnologías del yo y otros textos afines. Buenos Aires: Paidós.

20. Fridman, D. (2010). From Rats to Riches: Game Playing and the Production of the Capitalist Self. Special Issues on Knowledge in Practice, 423-226.

21. Fridman, D. (2016). Cashflow: Juego, autoayuda fnanciera y producción de sujetos económicos. Apuntes de Investigación del CECYP, 70.

22. G20 y OCDE. (2013). Advancing National Strategies for Financial Education, Joint Publication by Russia's G20 Presidency and the $O E C D$. Moscú: Internacional Gateway for Financial Education.

23. Garay, G. (2016). Índice de alfabetismo financiero, la cultura y la educación financiera. Perspectivas, núm. 37, mayo, 2016, pp. 23-40. 
24. Garcia, N., Grifoni , A., López, J. C., \& Mejía, D. M. (2013). La educación financiera en América Latina y el Caribe, situacion actual y perspectivas. Caracas, Venezuela: Cyngular.

25. Gómez, T., Molano, O. P., \& Rodríguez, S. (2015). La actividad lúdica como estrategia pedagógica para fortalecer el aprendizaje de los niños de la institución educativa Niño Jesús de Praga. Ibagué-Tolima: Universidad de Tolima.

26. González, P. (2012). Marcos y pruebas de evaluación de PISA 2012. España: OCDE.

27. Honohan, P. (2007). Cross-country variation in houshold acces to financial services. Washington D.C.: The World Bank.

28. Huang, W. H.-Y., \& Soman, D. (2013). A Practitioner's Guide to Gamification of Education. Toronto: Rotman School of Management.

29. INEE. (2013). Informe Español PISA 2012. Competencia financiera. Madrid: Instituto Nacional de Evaluación Educativa. Ministerio de Educación Cultura y Deporte.

30. INEGI. (2005). EEI-fichatecnica. Obtenido de EEI-fichatecnica: www.inegi.org.mx/rne/docs/Pdfs/Mesa5/20/EEI_fichatecnica.pdf

31. INEGI. (2010). Censo de poblacion y vivienda 2010. México: Sistema Nacional de Informacion Municipal .

32. Kiyosaki, R. T. (2012). Padre rico, padre pobre. Buenos Aires: Time $\&$ Money Network Editions.

33. Kiyosaki, R. T. (2015). El cuadrante del flujo del dinero. México: Aguilar.

34. Liu, C., Franklin, T., Shelor, R., Ozercan, S., Reuter, J., Ye, E., \& Moriaty, S. (2011). A Learning Game for Youth financial Literacy Education in the Teen Grid of Second Life Three-Dimensional Virtual Environment. merican Journal of Business Education, 8, 12-13.

35. Loungani, P. (2017). El crecimiento inclusivo y el FMI. Obtenido de Blog diálogo a fondo. Fondo Monetario Internacional (FMI): http://blog-dialogoafondo.imf.org/?p=7049

36. Marshall, E. (2011). Inclusión Financiera: Avances y Desafíos para Chile. Documentos de Política Económica del Banco Central de Chile, N. ${ }^{\circ} 41$ - Abril 2011.

37. Martínez-Carrasco, F., Muñoz, A. M., Eid, M., \& Colino, J. (2016). Inclusión financiera en el ámbito rural mediante cajas de ahorro. Estudio de una experiencia en México. Perfiles Latinoamericanos, núm. 48, julio-diciembre 2016, pp. 185-211.

38. Medina Riilla , A., \& Salador Mata, F. (2009). Didáctica General (2a ed.). (A. Cañizal, Ed.) Madrid, España: PEARSON. 
39. OCDE. (2005). Recommendation on Principles and Good Practices for Financial Education and Awareness. París: Organización para la Cooperación y el Desarrollo Económico.

40. OCDE. (2012). Marcos y Pruebas de Evaluación de PISA. En PISA 2012 Marco de Competencia Financiera (pág. 12). españa: OCDE.

41. OCDE. (2013). Marcos y pruebas de evaluación de PISA 2012. Competencia Financiera. París: Organización para la Cooperación y Desarrollo Económico.

42. OXFAM. (2017). Una economía para el 99\%. Oxford: OXFAM.

43. Pértega Díaz., S., \& Pita Fernández, S. (2004). Asociación de variables cualitativas: El test exacto de Fisher y el test de McNemar. Obtenido de

https://www.agamfec.com/antiga2013/pdf/CADERNOS/VOL11/VO L11_5/14_Invest_N11_5.pdf

44. Raccanello, K., \& Herrera, E. (2014). Educación e inclusión financiera. Revista Latinoamericana de Estudios Educativos (México), vol. XLIV, núm. 2, abril-junio 2014, pp.119-141.

45. Torres Maldonado , H., \& Girón Padilla , D. A. (2009). Didáctica General ( $1^{\mathrm{a}}$ ed., Vols. ISBN 978-9968-818-56-8). San José, C.R: Colección Pedagógica Formación Inicial de Docentes Centroamericanos de Educación Básica.

46. TRA Profesional Services. (2012). Secret to Achieving Financial Freedom. Obtenido de http://tra-professional.ie/wpcontent/uploads/2014/05/Financial-Freedom-workbook-Version-2.1010513-JC.pdf

47. Universidad Veracruzana. (s/f). Alfa de Cronbach y consistencia interna de un instrumento de medida. Obtenido de www.UVes/friasnav/AlfaCronbach.pdf

48. Vega Garnelo, A. (2013). La formación económico-financiera en Educación Secundaria Obligatoria en el marco de evaluación de la competencia financiera por PISA 2012. Trabajo Fin de Máster (pág. 20). Ponferrada: Universidad Internacional de la Rioja.

49. Velazco, M., \& Mosquera, F. (2010). Estrategias didácticas para el Aprendizaje Colaborativo PAIEP. Recuperado el 3 de Octubre de 2017, de Estrategias didácticas para el Aprendizaje Colaborativo PAIEP:

http://acreditacion.udistrital.edu.co/flexibilidad/estrategias_didacticas _aprendizaje_colaborativo.pdf

50. WEF. (2017). The Inclusive Growth and Development Report 2017. Geneva: World Economic Forum.

51. Yankovic, P. B. (21 de diciembre de 2010). El proyecto PISA y la competencia financiera (CF). Recuperado el 2017 de junio de 2017, de 
El proyecto PISA y la competencia financiera (CF): http://www.educativo.utalca.cl/medios/educativo/articulosydoc/finanz as.pdf 